
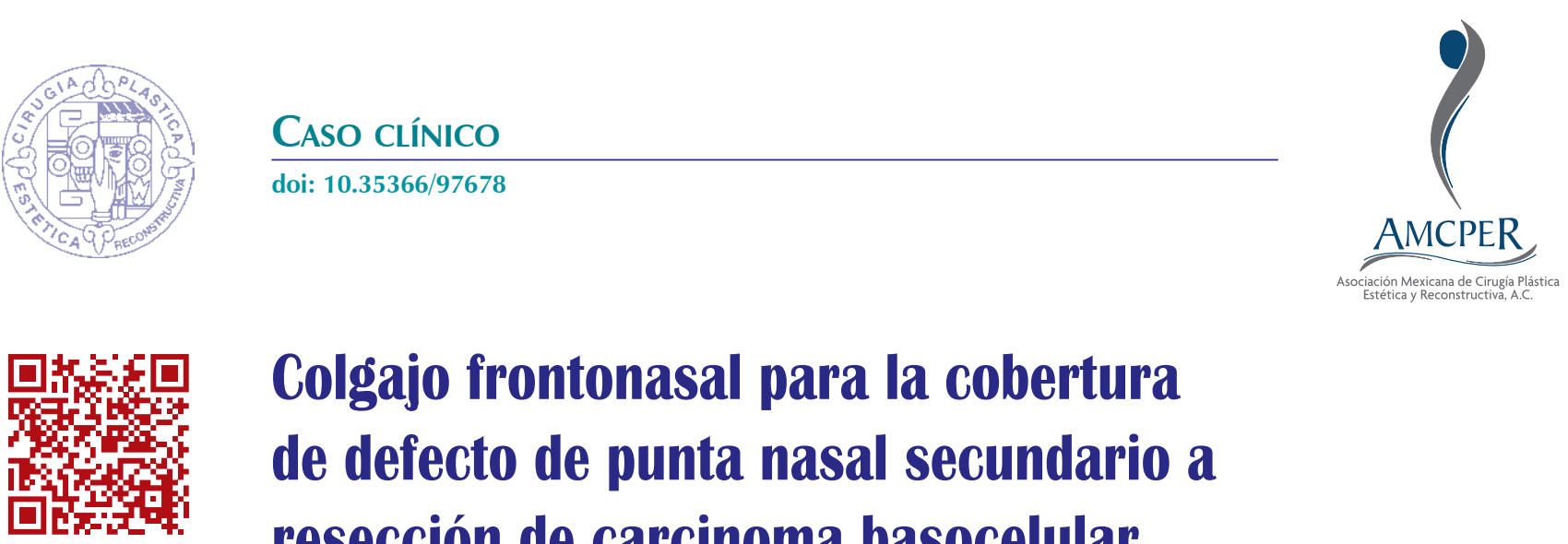

\title{
Colgajo frontonasal para la cobertura de defecto de punta nasal secundario a resección de carcinoma basocelular
}

\author{
Frontonasal flap for coverage of secondary nasal tip \\ defect resection of basal cell carcinoma \\ Dr. Ángel Alejandro Hernández-Moreno, * Dra. Hilda Alejandra Manzo-Castrejón, ${ }^{\ddagger}$ \\ Dr. Rodrigo Yahel Adame-Moreno ${ }^{\S}$
}

Palabras clave: Colgajo frontonasal, carcinoma basocelular, punta nasal, reconstrucción.

Keywords: Frontonasal flap, basal cell carcinoma, nasal tip, reconstruction.

\section{RESUMEN}

El colgajo frontonasal fue descrito por primera vez en 1967 por Rieger como un colgajo aleatorio de rotación para la cobertura de defectos de la punta nasal de hasta $2 \mathrm{~cm}$ de diámetro. Se trata de paciente masculino de 87 años con diagnóstico de carcinoma basocelular en la punta nasal, motivo por el cual fue intervenido para su resección y cobertura con colgajo frontonasal, con buena evolución y resultado. La reconstrucción de la punta nasal a partir del colgajo frontal tiene la ventaja de ofrecer mayor cobertura y menor retracción alar nasal así como un mejor resultado estético, pero tiene la desventaja de requerir como mínimo dos cirugías.

\section{ABSTRACT}

The frontonasal flap was first described in 1967 by Rieger as a random rotation flap for the coverage of nasal tip defects up to 2 centimeters in diameter. This was the case of 87-year-old male patient with a diagnosis of basal cell carcinoma in the nasal tip, which is why he underwent surgery for its resection and coverage with a frontonasal flap, with good evolution and results. Reconstruction of the nasal tip from the frontal flap has the advantage of offering greater coverage and less alar nasal retraction, as well as a better aesthetic result, but it has the disadvantage of requiring at least two surgeries.

\section{INTRODUCCIÓN}

$\mathrm{E}$ I colgajo frontonasal fue descrito por primera vez en 1967 por Rieger como un colgajo de rotación de tipo aleatorizado para la cobertura de defectos de la punta nasal de hasta $2 \mathrm{~cm}$ de diámetro. Su diseño inicial parte del borde superior del defecto describiendo una línea curva a través de la unión entre la pared nasal y la mejilla hasta alcanzar la glabela y descender hacia el canto interno contralateral, manteniendo una amplia base en una de las paredes laterales de la nariz. ${ }^{1}$
Los defectos nasales son a menudo el resultado de la eliminación de lesiones cutáneas. El cáncer de piel es la neoplasia más común y frecuente con más de un millón de casos en los Estados Unidos cada año; ocurre con mayor frecuencia en la cabeza y el cuello y la nariz es el subsitio más común. Aproximadamente 90\% de este tipo de cáncer es carcinoma de células basales, mientras que el resto es principalmente carcinoma de células escamosas y melanoma maligno. La escisión quirúrgica de Mohs es la técnica de tratamiento comúnmente preferida para el cáncer de piel, ya que ofrece una alta Servicios Sociales de los
Trabajadores del Estado (ISSSTE).

Recibido:

12 febrero 2020 Aceptado para publicar: 13 julio 2020
Citar como: Hernández-Moreno ÁA, Manzo-Castrejón HA, Adame-Moreno RY. Colgajo frontonasal para la cobertura de defecto de punta nasal secundario a resección de carcinoma basocelular. Cir Plast. 2020; 30 (2): 133-136. https://dx.doi.org/10.35366/97678 
eficacia de tratamiento (96-99\%), pero la cirugía en sí misma crea un defecto nasal. ${ }^{2}$

La reconstrucción de defectos quirúrgicos de la punta nasal y el tercio inferior de la nariz presenta un desafío único para el cirujano plástico. Las opciones de tratamiento incluyen: curación por segunda intención, injertos de piel de espesor total y colgajos locales o distantes. Debido a la textura y color únicos de la piel nasal, siempre es preferible que el tejido sea reemplazado por tejido similar. La reconstrucción debe adherirse a los principios de la subunidad nasal. ${ }^{3}$

Marchac y Toth diseñaron colgajos con un patrón vascular axial incorporando una rama constante de la arteria angular en el pedículo. ${ }^{4}$

Las posibles complicaciones en este tipo de colgajos incluyen el ensanchamiento del limbo debido a la tensión en el canto medial y la tracción no deseada en las áreas de la frente situadas medialmente. ${ }^{5}$

\section{CASO CLÍNICO}

Hombre de 87 años con diagnóstico probable de carcinoma basocelular en la punta nasal de características nodulares, de $1.3 \times 0.9 \mathrm{~cm}$, de color café grisáceo (Figura 1). Se realizó intervención quirúrgica por parte del servicio de oncología quirúrgica, con resección de la lesión

Figura 1:

Hombre de 87 años con lesión nodular en la punta nasal, probable carcinoma basocelular

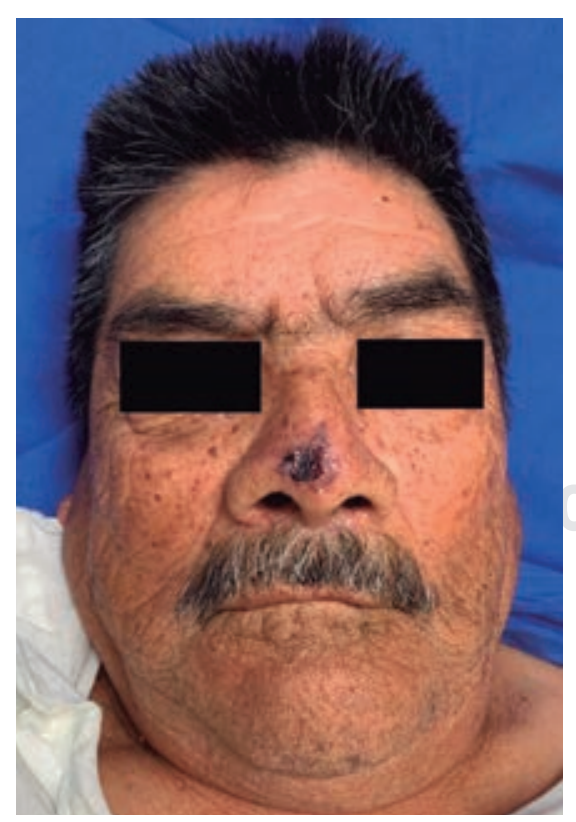

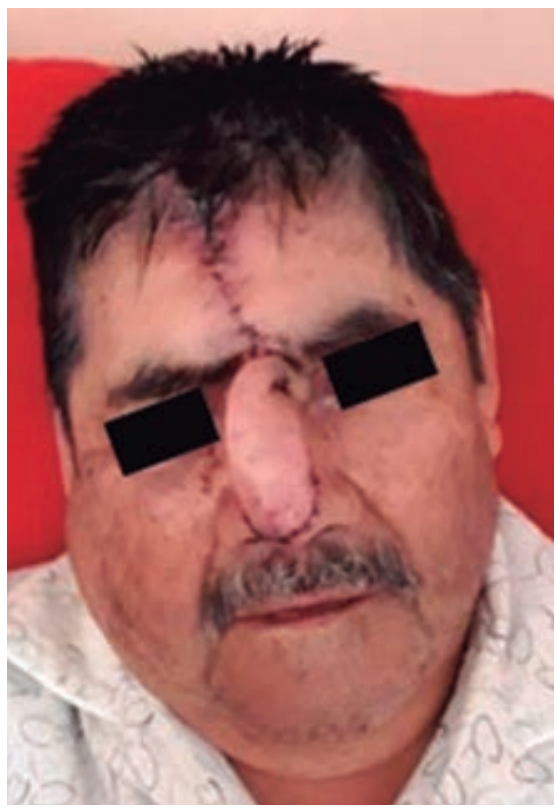

Figura 2: Paciente un día después del evento quirúrgico.

y en el mismo tiempo quirúrgico se reconstruyó por medio de un colgajo frontal medio.

\section{Técnica quirúrgica}

En el primer tiempo quirúrgico, previa resección de la neoplasia en la punta nasal, después de quedar un defecto de aproximadamente 2.4 $\times 2.4 \times 0.3 \mathrm{~cm}$, se trazó marcaje del colgajo con violeta de genciana en la región glabelar media, apoyándose en regla milimétrica estéril para lograr la cobertura total del defecto. Previa colocación de $10 \mathrm{~mL}$ de lidocaína al $2 \%$ y con latencia de 10 minutos, se realizó incisión con bisturí sobre sitio de marcaje, se inició en el lado derecho obteniendo un colgajo multilaminar de piel y tejido celular subcutáneo, preservando el músculo. Se elevó como un colgajo cutáneo con vascularización axial que se preserva al rotarlo y se fijó con nylon 6-0 puntos simples a los bordes del defecto. Se afrontó el área frontal en dos planos: el primero con poliglecaprone-25 de 3-0 puntos invertidos y el segundo con nylon 5-0 puntos intradérmicos. El reporte de patología del producto de resección de punta nasal fue carcinoma basocelular con patrón nodular con invasión a dermis reticular, con bordes negativos y lecho quirúrgico libre 
de tumor. El paciente fue egresado el mismo día con una adecuada coloración e hidratación del colgajo (Figura 2).

Fue revalorado en la consulta externa y tres semanas después programado para el segundo evento quirúrgico. En éste se procedió a resecar el colgajo, afrontando el borde libre del defecto con nylon 6-0 puntos simples y el área de pedículo con la misma técnica (Figura 3). El paciente continúa en control a través de consulta externa de cirugía plástica y reconstructiva, con una adecuada evolución y resultado (Figura 4).

\section{DISCUSIÓN}

El uso del excedente cutáneo de la región glabelar para la cobertura de los defectos del dorso nasal y del canto interno fue descrito en 1960 por McGregor, posteriormente, Rieger prolongó el diseño inicial hacia la totalidad del dorso nasal, siendo el primero en popularizar el colgajo frontonasal y su aplicación para la reconstrucción de defectos del tercio distal y de la punta nasal. La reconstrucción nasal postexéresis tumoral es una práctica habitual en pacientes añosos y pluripatológicos, en los

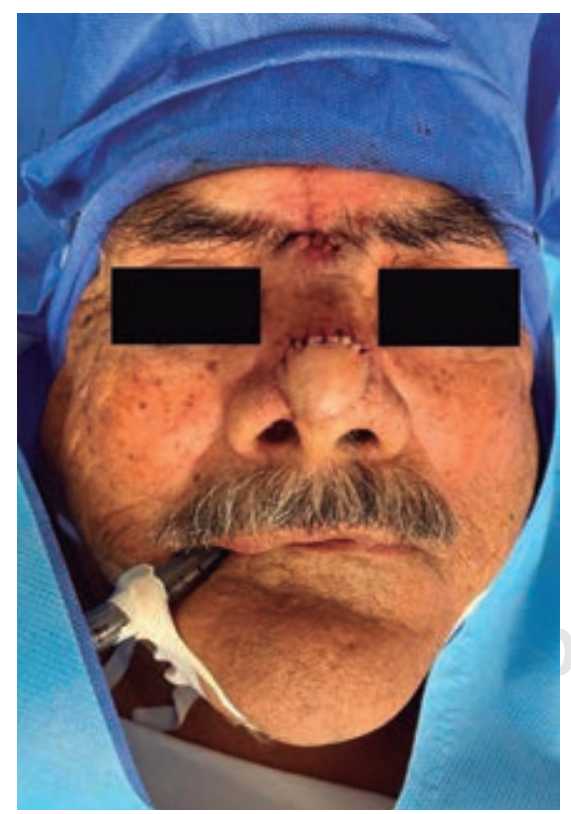

Figura 3: Paciente en el segundo evento quirúrgico tres semanas después con fijación y cierre del pedículo del colgajo.

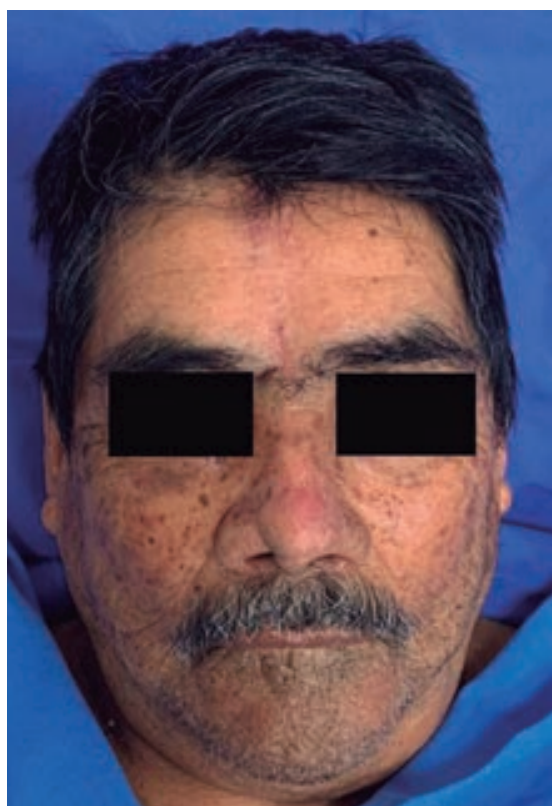

Figura 4: Paciente a las cuatro semanas del último evento quirúrgico.

que es fundamental optar por procedimientos quirúrgicos relativamente sencillos. ${ }^{1}$

El análisis minucioso de la deformidad visual, los principios regionales de la reconstrucción de la subunidad y la planificación cuidadosa determinan el éxito. ${ }^{6}$

Existen diferentes tipos de colgajos que se pueden utilizar en defectos nasales, por ejemplo: los colgajos bilobulados que con frecuencia presentan subunidades estéticas de tipo desfavorable y se mantienen visibles debido al edema prolongado con cicatrices casi circulares y base del colgajo estrecho. ${ }^{7}$

El colgajo Rintala es un colgajo de transposición miocutánea del dorso nasal que cubre defectos de la punta de tamaño mediano; sin embargo, crea cicatrices bilaterales, tiene un ancho limitado y no es adecuado para defectos que involucren subunidades adyacentes. En comparación, el colgajo frontonasal es más versátil al cubrir defectos de la punta y ala más anchos. El colgajo frontonasal paramediano sigue siendo el estándar de oro en defectos de la punta y ala grandes, pero habitualmente requiere dos o tres etapas quirúrgicas. ${ }^{8}$

El colgajo frontal tiene un resultado favorable tanto funcional como estético. Mureau y cola- 
boradores reportaron una satisfacción funcional en $81 \%$ de los pacientes, con resequedad de mucosa en $36 \%$, dificultad para el paso de aire en $31 \%$ y disminución del olfato en $16 \%$. Similitud de color de moderado a bueno en $97 \%$, crecimiento de pelo en 61 y $79 \%$ de los pacientes satisfechos con los resultados estéticos. Las complicaciones postoperatorias van de $1 \mathrm{a}$ $20 \%$, siendo las principales necrosis de la punta del colgajo en $7 \%$, estenosis de narina, retracción alar y resequedad de mucosa e infección. ${ }^{9,10}$

\section{CONCLUSIONES}

La reconstrucción de la punta nasal a partir del colgajo frontal tiene la ventaja de ofrecer mayor cobertura y menor retracción alar nasal así como un mejor resultado estético de la punta de la nariz. Tiene la desventaja de requerir como mínimo dos eventos quirúrgicos para obtener el resultado quirúrgico final y la necesidad de mantener un adecuado cuidado del pedículo del colgajo.

\section{REFERENCIAS}

1. Pérez M, Sancho J, Palao R, Barret JP. Aplicaciones del colgajo frontonasal para la cobertura de defectos nasales. Cir Plast Iberolatinoam 2015; 41 (4): 419-425. http://dx.doi.org/10.4321/S037678922015000400009.

2. Sapthavee A, Munaretto N, Toriumi DM. Skin Grafts vs Local Flaps for Reconstruction of Nasal Defects: A
Retrospective Cohort Study. JAMA Facial Plast Surg 2015; 17 (4): 270-273.

3. Zimbler MS, Thomas JR. The dorsal nasal flap revisited: aesthetic refinements in nasal reconstruction. Arch Facial Plast Surg 2000; 2 (4): 285-286.

4. Aoi J, Kajihara I, Masuguchi S, Fukushima S, Ihn $\mathrm{H}$. Simple and effective modification of the axial frontonasal flap to prevent flap distortion. J Dermatol 2019; 46 (1): 46-47.

5. Sanjuan-Sanjuan A, Ogledzki M, Ramirez CA. Glabellar flaps for reconstruction of skin defects. Atlas Oral Maxillofac Surg Clin North Am 2020; 28 (1): 43-48.

6. Menick FJ. Revising or redoing an imperfect or failed nasal reconstruction. Facial Plast Surg 2014; 30 (3): 342-356.

7. Helml G, von Gregory HF, Amr A, Fischer H, Gubisch $W$. One-stage nasal soft tissue reconstruction with local flaps. Facial Plast Surg 2014; 30 (3): 260-267.

8. Scheufler O. Variations in frontonasal flap design for single-stage reconstruction of the nasal tip. Plast Reconstr Surg 2016; 138 (6): 1032-1042.

9. Morales-Yépez HA, Erazo-Franco MA, ParadaVillavicencio $S$ et al. Reconstrucción nasal con colgajo frontal en un solo tiempo quirúrgico. Rev Sanid Milit Mex 2011; 65 (3): 125-130.

10. Mureau MA, Moolenburgh SE, Levendag PC, Hofer $\mathrm{SO}$. Aesthetic and functional outcome following nasal reconstruction. Plast Reconstr Surg 2007; 120 (5): 1217-1227.

\section{Correspondencia: \\ Dr. Ángel Alejandro Hernández Moreno \\ E-mail:dr.alexhmoreno@gmail.com}

Conflicto de intereses: Los autores de este artículo no tienen conflicto de intereses que declarar. 